\title{
Retro-odontoid Pseudotumor Associated with Sjögren Syndrome and Systemic Lupus Erythematosus Serology
}

SAEKO YAMADA, MD; YASUO NAGAFUCHI, MD, PhD; KEISHI FUJIO, MD, PhD; Department of Allergy and Rheumatology, Graduate School of Medicine, The University of Tokyo, Tokyo, Japan. Address correspondence to Dr. Y. Nagafuchi, Department of Allergy and Rheumatology, Graduate School of Medicine, The University of Tokyo, 7-3-1 Hongo, Bunkyo-ku, Tokyo, 113-8655, Japan. E-mail: nagafuchi-tky@umin.ac.jp. Keishi Fujio received financial support or fees from Astellas, BMS, MitsubishiTanabe, Eli Lilly, Pfizer, Santen, Takeda, Chugai, Eisai, Taisho Toyama, NihonKayaku, and UCB. Ethical approval not required for case reports, according to the policy of the University of Tokyo. Written informed consent was obtained from the patient to publish the material. J Rheumatol 2018;45:1424-5; doi:10.3899/jrheum.171251

We report here on the first case, to our knowledge, of retroodontoid pseudotumor (ROP) in a patient with an autoimmune disease other than rheumatoid arthritis (RA). It is possible that the onset of ROP was related to Sjögren syndrome (SS) with serology for systemic lupus erythematosus (SLE).

A 34-year-old man presented with an ROP without RA. Three years earlier, he had sicca symptoms and many renal and splenic infarctions, and was diagnosed with SS and antiphospholipid antibody syndrome. The anti-SSA, anti-SSB, anti-dsDNA, anti-RNP, and anti-Sm antibodies and lupus anticoagulant were positive, with low $\mathrm{C} 4$ level, meeting the European League Against Rheumatism/ American College of Rheumatology 2017 draft of the classification criteria for SLE. The patient was treated with warfarin. Recently he had occipital headaches; cerebrospinal fluid analysis and brain magnetic resonance imaging (MRI) were normal. A mass demonstrated on cervical MRI had the typical image of an ROP (Figure 1). We also suspected tension headaches. The pain was gradually alleviated with loxoprofen sodium.

ROP are occasionally caused by RA, atlantoaxial subluxation, amyloid deposits from dialysis, spondylosis, or ossification of the anterior longitudinal ligament. Degenerative changes in the cervical spine or ligament ossification cause immobility and instability of the cervical spines that result in pseudotumor formation ${ }^{1}$. No degenerative changes were apparent on dynamic radiograph or computed tomography images (Figure 2). Epidemiologically, ROP increase with $\mathrm{age}^{2}$. In cases without the aforementioned background, the youngest reported patient, based on our PubMed search, was 55 years old $^{3}$. Our patient was 34 years old; thus it was difficult to account for an ROP due to age alone.

There have been no ROP cases in patients with autoimmune diseases other than RA, to our knowledge. We report here the possibility that the onset of ROP was related to SS with SLE serology.

\section{REFERENCES}

1. Chikuda H, Seichi A, Takeshita K, Shoda N, Ono T, Matsudaira K, et al. Radiographic analysis of the cervical spine in patients with retro-odontoid pseudotumors. Spine 2009;34:E110-4.

2. Tojo S, Kawakami R, Yonenaga T, Hayashi D, Fukuda K. Factors influencing on retro-odontoid soft-tissue thickness: analysis by magnetic resonance imaging. Spine 2013;38:401-6.

3. Barbagallo GM, Certo F, Visocchi M, Palmucci S, Sciacca G, Albanese V. Disappearance of degenerative, non-inflammatory, retro-odontoid pseudotumor following posterior C1-C2 fixation: case series and review of the literature. Eur Spine J 2013;22 Suppl 6:S879-88.

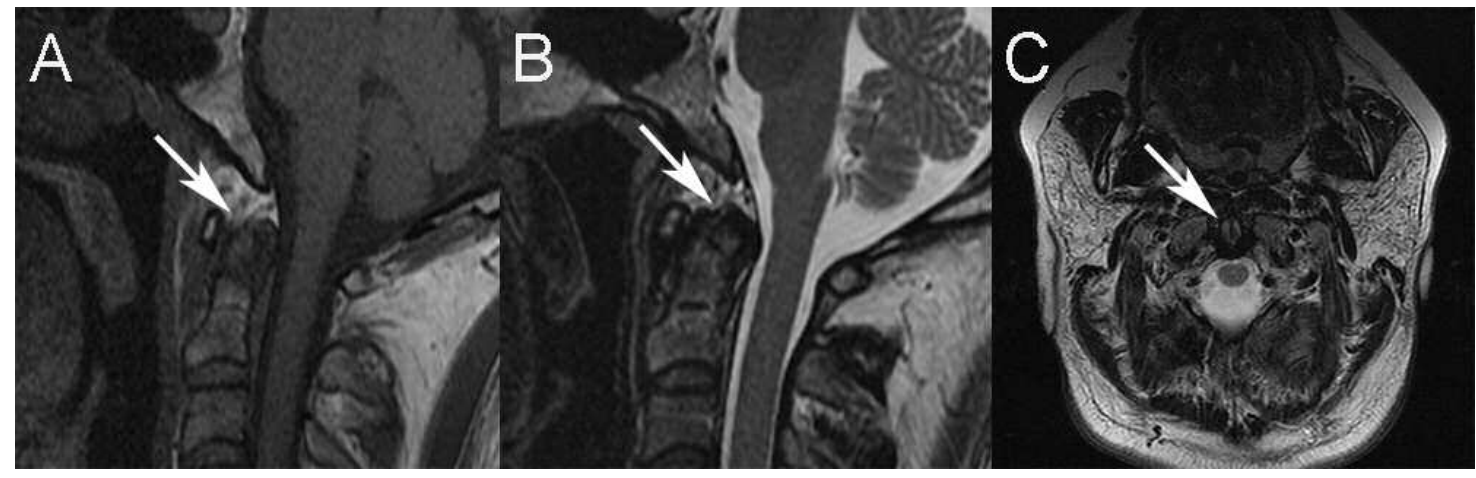

Figure 1. Retro-odontoid pseudotumor on cervical magnetic resonance image. A mass formation posterior to the odontoid process showed isointensity on a (A) mid-sagittal T1-weighted image, hypointensity on (B) mid-sagittal, and (C) axial T2-weighted images, and mild spinal canal narrowing.

Personal non-commercial use only. The Journal of Rheumatology Copyright @ 2018 . All rights reserved. 


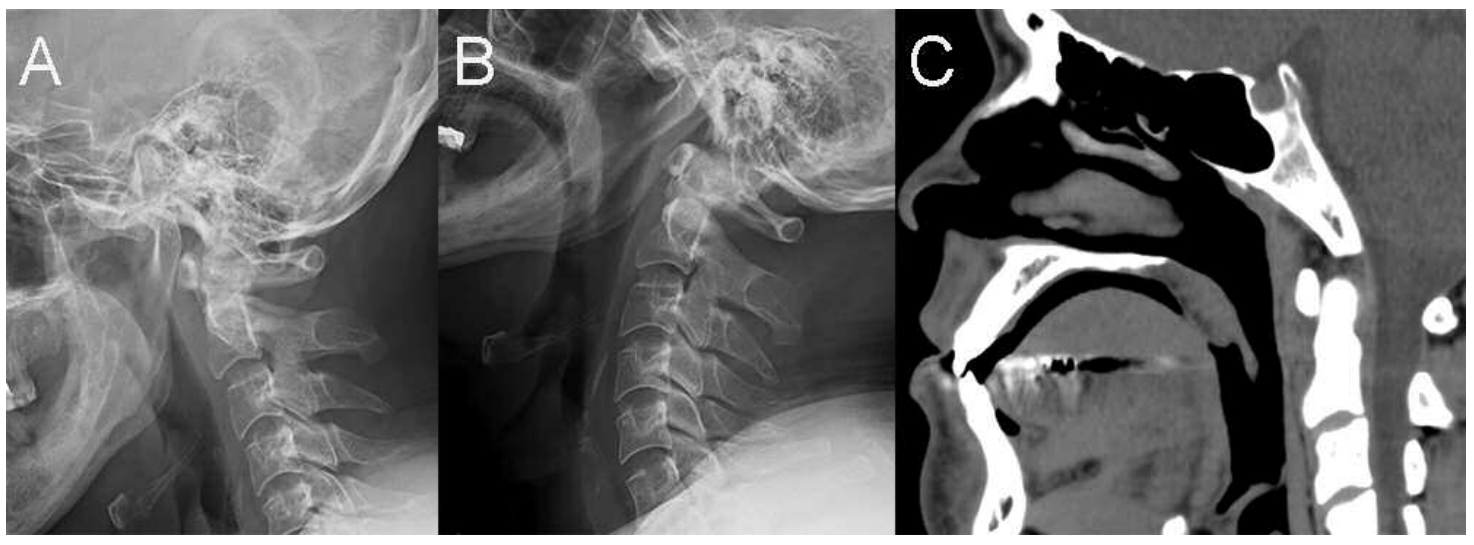

Figure 2. No degenerative changes were noted on dynamic radiograph and CT images. Cervical radiographic images during (A) flexion and (B) extension: the atlantodental interval was $2 \mathrm{~mm}$. (C) Mid-sagittal CT image showed no ossifications due to degenerative changes or ossification of the anterior longitudinal ligament. CT: computed tomography. 